\title{
Recent Results from the Antares Neutrino Telescope
}

\author{
Antoine Kouchner ${ }^{1, *}$ on behalf of the Antares Collaboration \\ ${ }^{1}$ AstroParticle and Cosmology (APC) \\ University Paris Diderot, CNRS/IN2P3, CEA/IRFU, Observatoire de Paris, Sorbonne Paris Cité, \\ 75205 Paris, France \\ Also at Institut Universitaire de France, 75005 Paris
}

\begin{abstract}
Antares, the first undersea neutrino telescope, has been continuously operating since 2007 in the Mediterranean Sea. The transparency of the water allows for a very good angular resolution in the reconstruction of neutrino events of all flavors. This results in an unmatched sensitivity for neutrino source searches, in a large fraction of the Southern Sky, at TeV energies. As a consequence, Antares provides valuable constraints on the origin of the cosmic neutrino flux discovered by the IceCube Collaboration. Based on an all-flavor dataset spanning nine years of operation of the detector, the latest results of Antares searches for neutrino point sources, and for diffuse neutrino emission from the entire sky as well as from several interesting regions such as the Galactic Plane, are presented. Several results have been obtained through a joint analysis with the IceCube Collaboration. Concerning the multi-messenger program, the focus is made on the follow-up searches of IceCube alerts, in particular the one related to the TXS $0506+056$ blazar, thought to be the first extragalactic high-energy neutrino source identified so far.
\end{abstract}

\section{Introduction}

The field of neutrino astronomy has made a major step forward with the detection, in 2013, of diffuse-like high-energy (HE) emission of neutrinos ( $>\mathrm{TeV}$ ) across the whole sky, by the IceCube experiment [1-3]. This major achievement opens the way towards a multi-messenger astronomy where neutrinos can be key players. They indeed carry unique information on the astrophysical processes occurring at sources, and since they are produced in a beam dumped scenario where hadrons must have been accelerated to high energies, they can reveal the longstanding question of the origin of ultra high-energy cosmic rays.

Since neutrinos only interact through the weak force, they can also probe denser media than other cosmic messengers such as gamma-rays. Along the same line, they can probe the universe over large distances, offering access to cosmological sources. This property turns at the same time into a detection challenge that can only be overcome through the instrumentation of km-scale detectors to compensate for the weaknesses of the interaction cross section and of the cosmic fluxes.

With the recent compelling identification by IceCube of the first extragalactic HE source namely the blazar TXS 0506+056 [4], triggered by a multi-messenger observation [5] -, active galactic nuclei (AGN [6]) have resurrected into ones of the most intriguing sources. But

\footnotetext{
*e-mail: kouchner@apc.univ-paris-diderot.fr
} 
transient sources, such as gamma ray bursts (GRB), remain of great interest and are carefully studies. High-energy gamma-rays $(>\mathrm{TeV}$ ) have also been observed from the central part of our galaxy, which hosts several classes of sources likely to produce hadrons and neutrinos such as supernovae remnants, microquasars, and even in certain models pulsar wind nebulae [7]. Galactic sources are the prime focus of Northern hemisphere telescopes like Antares. Indirect searches for Dark Matter using neutrinos are also pursued in Antares, from the Sun, the Earth and the Galactic Centre, for which Antares provides some of the best limits presently available. Details are given in re Ref [8] and are not addressed here.

Neutrino telescopes (NT) are gigantic open 3D arrays of photomultiplier tubes (PMTs) enclosed in pressure resistant glass spheres, referred to as optical modules (OMs). Relativistic particles are detected collecting the Cherenkov light induced by these particles in a transparent medium such as water or ice. In the search for neutrino interactions, atmospheric muons produced by the interaction of cosmic rays in the atmosphere are therefore the most abundant source of background. In order to mitigate it, the detectors are buried deep under the surface, and neutrinos are searched for as upgoing particles. Hence, NT are most efficiently monitoring one half of the sky in the TeV-PeV range. This restriction can be overpassed requiring a containment condition at the expense of a narrower energy range, focusing on the most energetic particles only. The interacting neutrinos produce two classes of events: tracks and cascades (also called showers). Track-like events are those where a long-ranged muon is observed, and allow for the precise measurement of its detection. This is the golden channel for astronomy. Most of these events are produced by charged-current interaction of muon neutrinos.

Cascades events originate from electromagnetic and hadronic showers induced by all-flavour neutrino interactions, and can be regarded as point-like sources of light in the detector. The Cherenkov light expands spherically from this bright point. In contrast to the tracks, cascades provide a poorer resolution on the direction of the incoming neutrinos. However, in sea water, the precision is good enough to search for small-scale sources in the Sky, thanks to the modest amont of light diffusion (comparatively to ice). In the case of cascades, the more localised distribution of light close to the neutrino interaction point allows for a better estimate of the neutrino energy, while in the case of track-like events, only a fraction of the energy deposited by the through-going muon can be seen.

\section{The Antares detector}

The Antares detector is located $40 \mathrm{~km}$ offshore from Toulon at a depth of $2475 \mathrm{~m}$ and comprises 12 mooring lines with 25 storeys housing 3 optical modules each. The Antares optical modules, hosting a large 10 -inch photomultiplier, are oriented at $45^{\circ}$ downward to be more sensitive to upward light fronts. A sketch of detector and detailed information about its technical configuration can be found in Ref [9].

\subsection{Long-term monitoring}

Several calibration methods have been developed in order to guarantee a proper time relative time resolution between the different components of the detector, in turn ensuring the expected reconstruction performance in term of angular accuracy (see section 4). Some of them take advantage of the presence in sea water of potassium whose isotope ${ }^{40} \mathrm{~K}$ decays producing electrons subsequently inferring Cherenkov light in the close vicinity of the OMs, at a rate of about $40 \mathrm{kHz}$ per OM. This light can be seem almost simultaneously by pairs of OMs of the same storeys. Studying these coincidences enable to correct for potential time offsets, but 

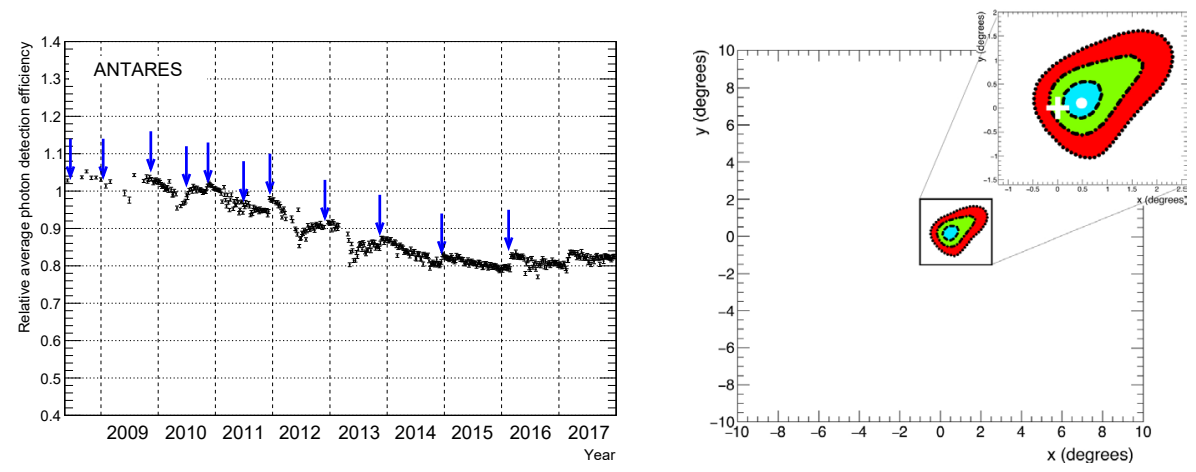

Figure 1. Left: Relative OM efficiency averaged over the whole detector against time. The blue arrows indicate the periods in which high voltage tunings of the PMTs has been performed, while error bars indicate the statistical error on the mean efficiency. Right: Moon shadow. Contour plots corresponding to different confidence levels $(68.27 \% ; 95.45 \% ; 99.73 \%)$. The dot represents the most significant position, while the cross indicates the nominal position of the Moon, i.e. the center of the FoV.

also provide a powerful tool to assess the evolution of the individual efficiencies of the OMs. Figure 1 (left panel) presents such evolution, averaged over the detector, as a function of time over almost of decade. The study [10] reveals a modest loss of about $20 \%$ only, which translates into an even smaller inefficiency when searching for $\mathrm{HE}$ neutrinos $(>\mathrm{TeV})$. The results of the study serve as input for the simulations, thus fairly reproducing the inefficiencies of each $\mathrm{OM}$ on a run-by-run basis.

\subsection{Absolute Pointing}

The proper measurement of the calibration constants, such as in the exemple above, allows inferring by Monte Carlo techniques the expected performances of the detector. However, the absolute pointing of the detector can hardly be assessed in absence of a well-known source of neutrinos. One way to overcome this limitation is to study downward going muons. Being produced by the interaction of very HE cosmic-rays in the atmosphere, those muons should exhibit a deficit in their intensity in the direction of the Moon which the parent cosmic rays by absorption. The study however requires a large statistics. Analysing the data between 2007 and 2016, the Moon shadow is detected with a statistical significance of $\sim 3.5 \sigma$ at a position consistent with expectation [11], as can been seen from the right panel of Figure 1.

\section{Search for a diffuse fluxes}

Despite the great intensity of the cosmic neutrino flux reported by the IceCube Collaboration, the size of the Antares detector remains too small for a high significance confirmation. Nevertheless, adding the cascade channel provides an additional sensitivity that permits to reach a rejection power as low as the IceCube measured flux, or constraint the hypotheses of dominant contribution from the Southern Sky, such as the central part of the Milky Way.

So far, 9 years of data have been used to search for an all sky cosmic neutrino flux concentrating on upward-going events to discard the atmospheric muon background, and including both track-like and cascade-like events [12]. After selection cuts, 33 events were observed, with $24 \pm 7$ being expected from pure background and about 8 from the astrophysical flux 

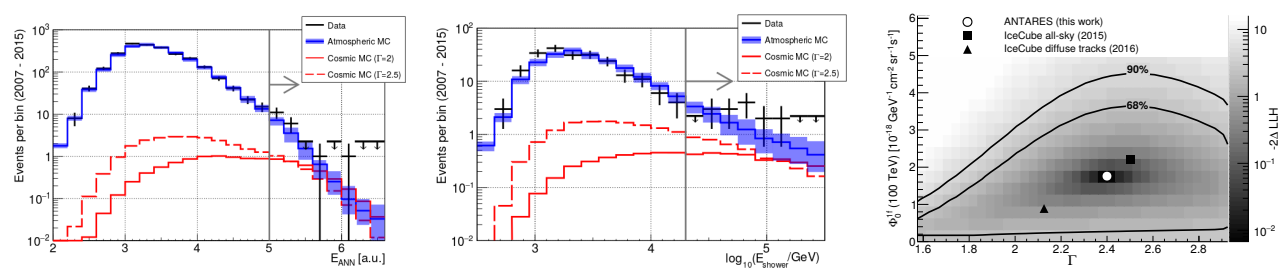

Figure 2. Distribution of the energy estimator for track-like (left panel) and shower-like (middle panel) events, after the event selection chain. The solid (dashed) red histogram shows the cosmic neutrino expectation for a cosmic flux proportional to $\mathrm{E}^{-2}\left(\mathrm{E}^{-2.5}\right)$ with normalization $\Phi_{0}^{1 f}(100 \mathrm{TeV})=10^{-18}$ $\left(1.5 \times 10^{-18}\right) \mathrm{GeV}^{-1} \mathrm{~cm}^{-2} \mathrm{~s}^{-1} \mathrm{sr}^{-1}$. The blue line represents the sum of all atmospheric events. The gray line represents the energy-related cut. Data after unblinding are shown as black crosses. Right: 2D log-likelihood scan of the diffuse cosmic flux normalization and spectral index obtained in the latest Antares search for a diffuse flux.

(Fig. 2, left and middle). This corresponds to a marginal excess of $1.6 \sigma$, but it may indicate a cosmic component. If interpreted so, the best fit for a single power-law cosmic neutrino spectrum (one flavor), at $100 \mathrm{TeV}$, is $\Phi_{0}^{1 f}(100 \mathrm{TeV})=(1.7 \pm 1.0) 10^{-18} \mathrm{GeV}^{-1} \mathrm{~cm}^{-2} \mathrm{~s}^{-1} \mathrm{sr}^{-1}$ with spectral index $2.4_{-0.4}^{+0.5}$ (Fig. 2, right).

Since a neutrino production is expected from the Galactic Plane due to the interaction of cosmic-rays with the Galactic gas and the radiation fields, it has been suggested that this specific region could significantly contribute to the measured IceCube flux. This hypothesis has recently been tested with ten years of Antares track and shower data, combined with a sample of seven years of IceCube tracks. The data are combined into a joint likelihood test for neutrino emission according to the recent models of Galactic CR diffusion, so-called $\mathrm{KRA}_{\gamma}$ models [13]. The analysis led to no significant excess [14] and the obtained limits start constraining the model parameter space, as can be seen in Figure 3.

\section{Search for point-like sources}

The latest searches for cosmic neutrino sources relies on a sample spanning between 2007 and the end of 2015 and making use of track- and shower-like events [15]. The sample consists of 7622 track events and 180 shower events. Four different searches have been performed: a scan over the whole Antares visible sky; an investigation of 106 pre-selected astrophysical candidates; a dedicated analysis of the Galactic Center (GC) region; and a study of Sagittarius A* investigated as a possible extended source. No evidence was found. Nevertheless, these searches provide the most sensitive limits for a large fraction of the Southern Sky (see Figure 3, right), in particular in the energy range relevant for Galactic sources $\left(E_{v}<100 \mathrm{TeV}\right)$. For the Southern Sky, a combination of the above-mentioned results and the ones from the IceCube Collaboration is presently ongoing, taking advantage of the complementarity of the samples. See Ref [16] for details.

\section{Search for transient phenomena}

Multi-messenger astrophysics aims at connecting traditional astronomy with charged cosmic ray, $\gamma$-ray, gravitational wave and neutrino observations. The Antares Collaboration participates in this global efforts through various data exchange agreements. Among all the possible 

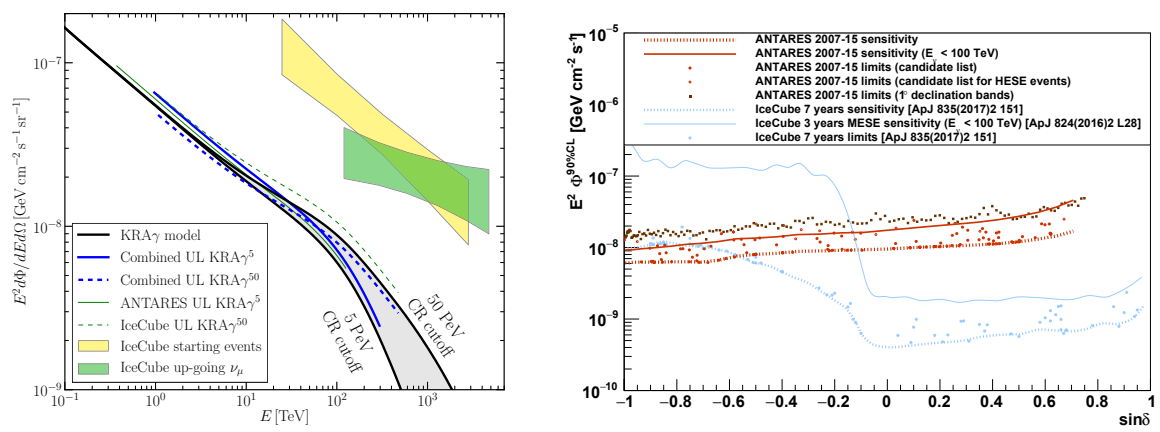

Figure 3. Left: Combined upper limits (UL) at $90 \%$ C. L. (blue lines) on all-flavor neutrino flux of the $\mathrm{KRA}_{\gamma}$ model with the 5 and $50 \mathrm{PeV}$ cutoffs (black lines), see Ref [13] for explanations. The boxes represent the diffuse astrophysical neutrino fluxes measured by IceCube using an isotropic flux template with starting events (yellow) and upgoing tracks (green). Right: Sensitivities (lines) and upper limits at 90\% C.L. (red circles) on one-flavor from the investigated candidates assuming an $E^{-2}$ spectrum.

astrophysical sources, transient ones are the most promising because of the increased observation possibilities offered by the suppression of the atmospheric background in the welldefined space-time window provided by partners.

Of the most salient examples, besides the observations based on Gravitational Waves [17], is the successful identification of the blazar TXS 0506+056 after the IceCube alert (IC$170922 \mathrm{~A}$ ) could be connected to observations in $\gamma$-rays and at larger wavelengths $[4,5]$.

The Antares Collaboration also searched for neutrino candidates in the direction of TXS $0506+056$, following three approaches. The first study refers to the online follow-up of the IceCube alert, which yields a null result. The second search is based on the standard time-integrated reported in section 4, extended up to 2017 specifically for this source. The analysis fits 1.03 signal events in the direction of TXS 0506+056. The corresponding to a p-value (no trials accounted) is $3.4 \%$, making it the candidate with the third smallest p-value in our list. Finally, the third strategy uses the information from the IceCube time-dependent analysis reporting a bursting activity centred on December 13, 2014, as input for an Antares time-dependent analysis. No correlated events are found. Details on the three approaches are available in Ref [18].

\section{Updated Oscillation Studies}

Antares was the first Neutrino Telescope to provide an evidence for neutrino oscillations in the atmospheric sectors [19]. This first study was based on a data sample recorded between 2007 and 2010. With the larger sample available by now, this study was updated and improved. A new measurement of $\Delta m_{32}^{2}$ and $\theta_{23}$ has been performed including a larger set of systematics, three of them being related to the atmospheric neutrino flux (see Ref [20] for details). The results of the final fit are depicted in Figure 4. Though not competitive, they are consistent with world best-fit values. The same sample have been used to test the hypothesis of the existence of a fourth family of neutrinos. The exclusion plot shown in Figure 4 (right) reveals the best constraint in the word for a some region of the parameter space. 

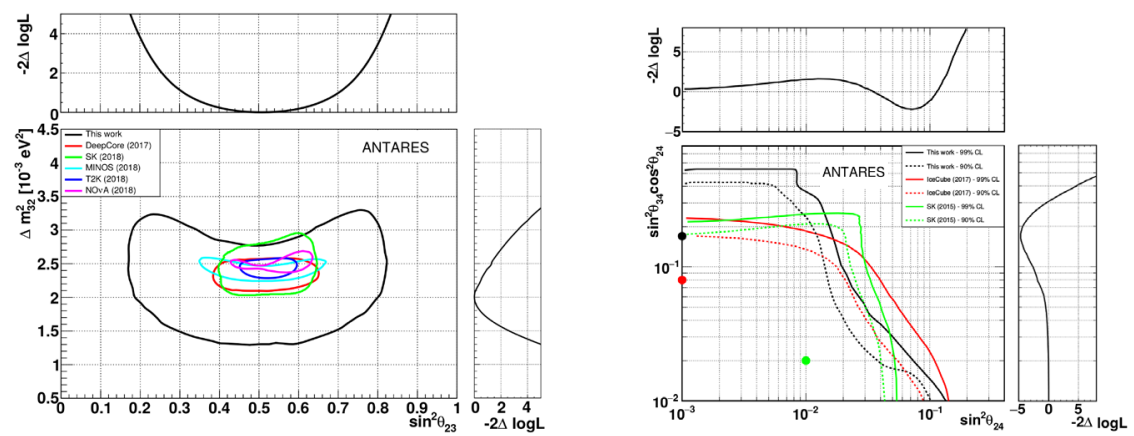

Figure 4. Left: Antares contour at $90 \% \mathrm{CL}$ in the plane of $\sin ^{2} \theta_{23}$ and $\Delta m_{32}^{2}$ (black line) compared to the results by several other experiments. Right: $90 \%$ (dashed lines) and $99 \%$ (solid lines) CL limits for the $3+1$ neutrino model in the parameter plane of $\left|U_{\mu 4}\right|^{2}=\sin ^{2} \theta_{24}$ and $\left|U_{\tau 4}\right|^{2}=\sin ^{2} \theta_{34} \cos ^{2} \theta_{24}$ obtained with respect to the non-sterile hypothesis (black lines) and compared to the other results.

\section{Conclusions}

Antares has been taking data for more than a decade, demonstrating the potential for very large deep-sea neutrino telescopes with unmatched angular accuracy, and paved the way for KM3NeT, the next-generation of detector being constructed in the Mediterranean. The physics results obtained place valuable constraints on the origin of the IceCube astrophysical signal. The operation should end in 2020 with the recovery of the detector components.

\section{References}

[1] M.G. Aartsen et al., Phys. Rev. Lett. 113: 101101 (2014).

[2] M.G. Aartsen et al., Astrophys. J. 809: 98 (2015).

[3] M.G. Aartsen et al., Astrophys. J. 833: 3 (2016).

[4] M.G. Aartsen et al. Science 361, 147-151 (2018)

[5] IceCube et al., Science 361, eaat1378 (2018)

[6] A. Fedynitch, this workshop.

[7] M. Kachelriess, this workshop.

[8] J.A Aguilar Sanchez, this workshop ; S. Gozzini, this workshop.

[9] M. Ageron et al., Nucl. Inst. Meth. A 65611 (2011) .

[10] A. Albert et al., Eur. Phys. J. C 78669 (2018).

[11] A. Albert et al., Eur. Phys. J. C 781006 (2018).

[12] A. Albert et al., ApJL 853 L7 (2018).

[13] D. Gaggero et al., ApJL 815 L25 (2015).

[14] A. Albert et al., ApJL 868 L20 (2018).

[15] A. Albert et al., Phys. Rev. D 96 082001(2017).

[16] G. Illuminati, this workshop.

[17] C. Finley, this workshop.

[18] A. Albert et al., ApJL 863 L2 (2018).

[19] S. Adrian-Martinez et al., Phys. Lett. B 714224 (2012) .

[20] A. Albert et al., https://arxiv.org/abs/1812.08650. 